\title{
Evaluation of Interferon Resistance in Newly Established Genotype 1b Hepatitis C Virus Cell Culture System
}

\author{
Miki Taniguchi ${ }^{1 \dagger}$, Megumi Tasaka-Fujita ${ }^{1,2 \dagger}$, Mina Nakagawa ${ }^{1,2}$, Takako Watanabe ${ }^{1,4}$, \\ Fukiko Kawai-Kitahata ${ }^{1}$, Satoshi Otani ${ }^{1}$, Fumio Goto ${ }^{1}$, Hiroko Nagata ${ }^{1}$, Shun Kaneko ${ }^{1}$, \\ Sayuri Nitta ${ }^{1}$, Miyako Murakawa ${ }^{1}$, Yuki Nishimura-Sakurai ${ }^{1}$, Seishin Azuma ${ }^{1}$, Yasuhiro Itsui ${ }^{1,4}$, \\ Kenichi Mori ${ }^{5}$, Shintaro Yagi ${ }^{5}$, Sei Kakinuma ${ }^{1,3}$, Yasuhiro Asahina ${ }^{1,3^{*}}$ and Mamoru Watanabe ${ }^{1}$ \\ ${ }^{1}$ Department of Gastroenterology and Hepatology, Tokyo Medical and Dental University, Tokyo, Japan; ${ }^{2}$ Center for \\ Interprofessional Education, Tokyo Medical and Dental University, Tokyo, Japan; ${ }^{3}$ Department of Liver Disease Control, Tokyo \\ Medical and Dental University, Tokyo, Japan; ${ }^{4}$ Department of Medical Education Research and Development, Tokyo Medical and \\ Dental University, Tokyo, Japan; ${ }^{5}$ Advanced Life Science Institute, Saitama, Japan
}

\begin{abstract}
Background and Aims: The hepatitis C virus (HCV) genotype $1 \mathrm{~b}$ is known to exhibit treatment resistance with respect to interferon (IFN) therapy. Substitution of amino acids 70 and 91 in the core region of the $1 \mathrm{~b}$ genotype is a significant predictor of liver carcinogenesis and poor response to pegylated-IFN- $\alpha$ and ribavirin therapy. However, the molecular mechanism has not yet been clearly elucidated because of limitations of the HCV genotype $1 \mathrm{~b}$ infectious model. Recently, the TPF1-M170T HCV genotype 1b cell culture system was established, in which the clone successfully replicates and infects Huh-7-derived Huh7-ALS32.50 cells. Therefore, the purpose of this study was to compare IFN resistance in various $\mathrm{HCV}$ clones using this system. Methods: HCV core amino acid substitutions R70Q and L91M were introduced to the TPF1-M170T clone and then transfected into Huh7-ALS32.50 cells. To evaluate the production of each virus, intracellular HCV core antigens were measured. Results were confirmed with Western blot analysis using anti-NS5A antibodies, and IFN sensitivity was subsequently measured. Results: Each clone was transfected successfully compared with $\mathrm{JFH}-1$, with a significant difference in intracellular HCV core antigen $(p<0.05)$, an indicator of continuous HCV replication. Among all clones, L91M showed the highest increase in the HCV core antigen and HCV protein. There was no significant resistance against IFN treatment in core substitutions; however, IFN sensitivity was significantly different between the wildtype core and JFH-1 $(p<0.05)$. Conclusions: A novel genotype $1 \mathrm{~b} \mathrm{HCV}$ cell culture was constructed with core amino acid substitutions, which
\end{abstract}

Keywords: Cell culture system; Core Amino acid substitutions 70 and 91; Genotype 1b; Hepatitis C virus.

Abbreviations: $\mathrm{Ag}$, antigen; CLIA, chemical reaction immunoassay; HCV, hepatitis $C$ virus; IFN, interferon; PBS, phosphate-buffered saline; SNP, single nucleotide polymorphism; SVR, sustained virologic response; WT, wildtype.

Received: 06 December 2015; Revised: 26 December 2015; Accepted: 28 December 2015

DOI: $10.14218 /$ JCTH.2015.00047.

$\dagger$ These authors contributed equally to this work.

* Correspondence to: Yasuhiro Asahina, Department of Gastroenterology and Hepatology, Tokyo Medical and Dental University, 1-5-45 Yushima, Bunkyo-ku, Tokyo 113-8519, Japan. Tel: +81-358035877, Fax: +81-358030268, E-mail: asahina.gast@tmd.ac.jp demonstrated IFN resistance of genotype $1 \mathrm{~b}$. This system will be useful for future analyses into the mechanisms of HCV genotype $1 \mathrm{~b}$ treatment.

(C) 2016 The Second Affiliated Hospital of Chongqing Medical University. Published by XIA \& HE Publishing Inc. All rights reserved.

\section{Introduction}

Hepatitis C virus (HCV) infection is the major cause of chronic liver diseases that lead to cirrhosis and hepatocellular carcinoma. An estimated $130-170$ million people worldwide are chronically infected with $\mathrm{HCV}^{1}$ Furthermore, HCV infection has been implicated in $28 \%$ of cirrhosis cases and $26 \%$ of liver cancer cases each year; thus, accounting for almost 500,000 deaths per year globally. ${ }^{2,3}$

Previous HCV therapies comprised interferon (IFN)-based regimens, such as pegylated-IFN- $\alpha$ and ribavirin, for up to 72 weeks, which lead to sustained virologic response (SVR) rates in approximately $50 \%$ of compliant patients infected with $\mathrm{HCV}$ genotype 1.4 Among the direct-acting antiviral agents, the first generation NS3/4A protease inhibitor telaprevir, in combination with pegylated-IFN- $\alpha$ and ribavirin, has been approved since 2011 for the treatment of patients infected with HCV genotype 1 . Increasing cure rates of approximately $70 \%$ have been achieved; however, adverse events of telaprevir were frequent, sometimes severe, and in some cases, treatment limiting. ${ }^{5}$ In 2014 , the first IFNfree and all-oral regimen combined with NS3/4A protease and an NS5A inhibitor was initiated in Japan. ${ }^{6}$ This phase 3 trial reported that all-oral regimens were well tolerated with a low incidence of serious adverse effects and high SVR rates within the range of $80-90 \%$. This early evidence suggests that combination therapy with direct-acting antiviral agents and without IFN may offer fewer side effects and higher SVR rates, but also may introduce issues on affordability and accessibility, including the emergence of drug-resistant HCV mutants and hepatocarcinogenesis after achieving SVR. ${ }^{7}$ Therefore, treatment with IFN in combination with direct-acting antiviral agents is still of great value because of its cost effectiveness, lower drug-drug 
Taniguchi M. et al: IFN resistance in $\mathrm{HCV}$ in vitro

interactions, lower emergence of resistant mutants, and suppressive effect of carcinogenesis.

Molecular studies on HCV have been hampered by the lack of efficient in vitro and in vivo models of infection, which have been partly overcome by the development of HCV subgenomic replicons $\mathrm{s}^{8,9}$ and an HCV-JFH1 genotype $2 \mathrm{a}$ cell culture system. ${ }^{10}$ Recent developments have led to the establishment of efficient culture-adapted full-length systems for isolates of genotypes $1 \mathrm{a}$ (strain TN), ${ }^{11} 2 \mathrm{a}$, and $2 \mathrm{~b}$, but have never led to the generation of a robust genotype $1 \mathrm{~b}$ system. ${ }^{12}$

Our previous study described viral infection, replication kinetics, and drug resistance of HCV core mutants using the $\mathrm{HCV}-\mathrm{JFH} 1$ cell culture system. ${ }^{13}$ With this system, IFN resistance of HCV core amino acid 70/91 substitutions could be determined by cellular expression levels of interleukin- 6 and upregulation of SOCS3 that lead to the suppression of the JAK/STAT pathway. However, this system was only established for genotype $2 a$, which is different from the clinically reported IFN-resistant genotype $1 \mathrm{~b}$. In the clinical setting, pathology and treatment responses in chronic hepatitis $\mathrm{C}$ vary among genotypes. ${ }^{14}$

Recently, a genotype $1 \mathrm{~b}$ HCV cell culture system, TPF1M170T, has been established (data in submission). The strain was cloned from a patient with HCV-related cirrhosis who developed fibrosing cholestatic hepatitis after liver transplantation. In this system, replication-enhancing mutations were introduced into the NS2 and NS4B regions to enable abundant replication. Simultaneously, Huh7-ALS32.50 cells were cloned as one of the most adapted Huh7 cell lines for the TPF1-M170T strain. The cell line was established by treating replicon-transfected Huh7 cells with IFN- $\alpha$ A/D.

The aim of the present study was to compare IFN sensitivity in various clones, including genotype 1 and 2 with or without core amino acid substitutions that are clinically important for treatment resistance and liver carcinogenesis. ${ }^{15,16} \mathrm{~A}$ novel HCV cell culture system was established by introducing core amino acid 70/91 substitutions in genotype $1 \mathrm{~b}$. Virus replication and production, and IFN sensitivity were subsequently evaluated.

\section{Materials and methods}

\section{Reagents and antibodies}

Recombinant human IFN- $\alpha-2 b$ was obtained from ScheringPlough (Kenilworth, NJ, USA). Antibodies used were: HCV core (Abcam, Cambridge, UK), NS5A (BioDesign, Saco, ME, USA), and $\beta$-actin (Sigma-Aldrich, St. Louis, MO, USA). Secondary antibodies were peroxidase-labeled anti-mouse (GE Healthcare; Little Chalfont, UK) and Alexa Fluor 488labeled goat anti-mouse (Invitrogen of Thermo Fisher Scientific, Waltham, MA, USA) IgG antibodies.

\section{Cell lines and culture conditions}

Huh7-ALS32.50 cells were maintained in Dulbecco's modified medium (Sigma-Aldrich) supplemented with $10 \%$ fetal bovine serum, $100 \mathrm{IU} / \mathrm{mL}$ penicillin, $100 \mu \mathrm{g} / \mathrm{mL}$ streptomycin (Nacalai Tesque, Kyoto, Japan), and non-essential amino acids (Gibco of Thermo Fisher Scientific) at $37^{\circ} \mathrm{C}$ under $5 \%$ $\mathrm{CO}_{2}$. Cells were maintained at a confluence range of $60-70 \%$. For culture passages, cells were incubated in trypsin with $0.05 \%$ EDTA at $37^{\circ} \mathrm{C}$ for $4 \mathrm{~min}$ for cell detachment; the cells were then rinsed 4-5 times with the culture medium to prevent further enzymatic degradation due to trypsin exposure. The cells were collected in a conical tube and then subjected to low-speed centrifugation at $700 \mathrm{rpm}$ for $5 \mathrm{~min}$ at $4^{\circ} \mathrm{C}$ to isolate the pellet. The supernatant was discarded, and the cells were resuspended in fresh culture media. HCV RNA transcribed from pTPF1-M170T and pJFH-1 was transfected into Huh7-ALS32.50 cells.

\section{Introduction of core amino acid substitutions}

Based on the PTPF1-M170T (GenBank Accession Number: LC011929), which is a 12,526 bp plasmid, full-length $1 \mathrm{~b}$ clones were constructed with core amino acid mutations that are clinically known for their treatment resistance. A fulllength PTPF1-M170T was digested with restriction enzyme (NotI; New England Biolabs, Ipswich, MA, USA), and the DNA fragments encompassing the full sequence of the core region were subcloned into pcDNA3.1. Mutations were introduced into the DNA fragment of the subcloning vector using a Quickchange II XL site-directed mutagenesis kit (Agilent Technologies, Santa Clara, CA, USA). Finally, these mutants were sequenced (Hokkaido System Science, Hokkaido, Japan).

The pTPF1-M170T plasmids, which encode the full-length genotype1b sequence, were linearized at their $3^{\prime}$ ends and used as templates for HCV RNA synthesis using the RiboMax large-scale RNA production system (Promega, Madison, WI, USA). After undergoing DNaseI treatment (RQ-1 RNase-free DNase; Promega), the transcribed HCV RNA was purified using ISOGEN reagent (Nippon Gene, Tokyo, Japan).

\section{Electroporation}

For RNA transfection, Huh7-ALS32.50 cells were cultured for 7-14 days after thawing to $90 \%$ confluence. The cells were washed twice in phosphate-buffered saline (PBS); $4 \times 10^{6}$ cells were then suspended in cytomix $(120 \mathrm{mM} \mathrm{KCl}$, $0.15 \mathrm{mM} \mathrm{CaCl}, 10 \mathrm{mM} \mathrm{K} \mathrm{HPO}_{4} / \mathrm{KH}_{2} \mathrm{PO}_{4}[\mathrm{pH} 7.6], 25 \mathrm{mM}$ HEPES [pH 7.6], 2 mM EGTA [pH 7.6], $5 \mathrm{mM} \mathrm{MgCl}$, filtered) containing $10 \mu \mathrm{g}$ of HCV RNA. These cells were transferred into a $4 \mathrm{~mm}$ electroporation cuvette (Gene Pulser Cuvette; Bio-Rad Laboratories, Inc., Hercules, CA, USA) and subjected to an electric pulse ( $250 \mathrm{~V}$ and $950 \mathrm{~F}$ ) using a Gene Pulser Xcell (Bio-Rad Laboratories, Inc.). These transfected cells were slowly added to $1 \mathrm{~mL}$ of culture medium placed in a cuvette and were left to stand for $15 \mathrm{~min}$ at room temperature, followed by incubation at normal culture conditions. Approximately $24 \mathrm{~h}$ post-transfection, the cells were washed twice in PBS and then incubated with the new culture medium, which was replaced every other day.

\section{Quantification of intracellular HCV core antigen (Ag)}

Transfected cells in each culture dish were lysed in $100 \mu \mathrm{L}$ lysis buffer (10 mM Tris- $\mathrm{HCl}[\mathrm{pH} 7.4], 0.15 \mathrm{M} \mathrm{NaCl}, 1 \mathrm{mM}$ EDTA, $0.5 \%$ NP40, $0.1 \%$ SDS, $0.04 \% 1 \times$ cOmplete Mini: Rishe Diagnostics, Tokyo, Japan). Next, cells were sonicated for 10 min (Biorupter UCW-201; BM Equipment Co., Tokyo, Japan) and centrifuged; the resulting supernatant was aspirated from these lysates and $270 \mu \mathrm{L}$ fetal calf serum was added to $30 \mu \mathrm{L}$ of each sample liquid. Samples were stored at $-80^{\circ} \mathrm{C}$ and measured using a chemical reaction immunoassay (CLIA) following the manufacturer's protocol (SRL, Tokyo, Japan). 
Taniguchi M. et al: IFN resistance in $\mathrm{HCV}$ in vitro

Identification of IL28B single nucleotide polymorphisms (SNPs) in Huh7-ALS32.50 cells

Identification of IL28B SNPs (rs8099917, rs11881222, and rs8103142) in Huh7-ALS32.50 cells was performed using an invader assay, following the manufacturer's protocol (SRL, Tokyo, Japan).

\section{RNA extraction, CDNA synthesis, and real-time PCR}

HCV-transfected Huh7-ALS32.50 cells were cultured with various concentrations of IFN- $\alpha-2 b$, such that the final DMSO concentration was < $1 \%$. For HCV detection, RNAs were isolated using an RNeasy Mini Kit (Qiagen, Hilden, Germany); the concentration of RNA products was determined using a NanoDrop 1000 spectrophotometer (Thermo Fisher Scientific). Total cellular RNA was used to generate cDNA from each sample using SuperScript II reverse transcriptase (Invitrogen). mRNA expression levels were quantified using the Taq Man Universal Master Mix II, no UNG (Applied Biosystems of Thermo Fisher Scientific) and the StepOnePlus Real-Time PCR System (Applied Biosystems).

The primers used for genotype $1 \mathrm{~b}$ core were: sense, $5^{\prime}$ GGT ACT GCC TGA TAG GGT GCT T-3', and antisense, 5'-TGG TIT TTC TIT GAG GTT TAG GAT TC-3'. The amount of HCV RNA was normalized to GAPDH using huGAPDH probe dye (Applied Biosystems). Assays were performed in triplicate, and the results are expressed as mean percentage of the control \pm SD. The $50 \%$ effective concentration $\left(E_{50}\right)$ for each compound was calculated three times using the probit method and expressed as mean \pm SD.

\section{Western blot analysis}

Western blot was performed as previously described. ${ }^{17,18}$ Briefly, $10 \mu \mathrm{g}$ of total cell lysate was separated using NuPAGE4-12\% Bis-Tris Midi Gel (Life Technologies of Thermo Fisher Scientific) and then blotted onto a polyvinylidene fluoride membrane (Roche Holding AG Basel, Switzerland). The membrane was incubated with the primary antibodies for HCV core and NS5A followed by a peroxidase-labeled antiIgG antibody; the results were visualized by chemiluminescence using the ChemiDoc MP Imaging System (Bio-Rad Laboratories, Inc.).

\section{Immunohistochemistry}

HCV-transfected Huh7-ALS32.50 cells were cultured on 22mm-round micro cover glasses (Matsunami, Tokyo, Japan), which were coated with $0.1 \%$ gelatin. For the detection of endoplasmic HCV core protein, cells were fixed with $4 \%$ paraformaldehyde for $10 \mathrm{~min}$ and permeabilized with $1 \%$ PBS-Tween for $10 \mathrm{~min}$. The cells were incubated overnight at $4^{\circ} \mathrm{C}$ with the primary antibodies. The fluorescent secondary antibodies were Alexa Fluor 488 goat anti-mouse IgG. The cells were mounted with a Vectashield mounting medium with DAPI (Vector Laboratories, Burlingame, CA, USA) and visualized using a confocal laser-scanning microscope (FV10i; Olympus Corp., Tokyo, Japan).

\section{Statistical analysis}

Statistical analyses were performed by Welch's $t$ test, with $p<0.05$ considered as statistically significant.

\section{Results}

\section{Introduction of core amino acid 70/91 substitutions to TPF1-M170T}

The IL28B SNPs (rs8099917, rs11881222, and rs8103142) of Huh7-ALS32.50 cells were all treatment-resistant heterozygous genotypes (TG, AG, and TC, respectively). The following new clones were constructed with core amino acid substitutions: Core wildtype, R70Q, L91M, and R70QL91M. R70Q and L91M (Fig. 1a), which were reported as clinically resistant to pegylated-IFN- $\alpha$ and ribavirin therapy. ${ }^{17}$ The R70Q strain is the same as the PTPF1-M170T, which harbors an R70Q mutation.

Immunohistochemistry was performed to clarify the transfection efficiency of these mutants. The transfected cells were fixed $72 \mathrm{~h}$ after electroporation, and the infected foci were visualized by immunostaining against core antibodies. All cells in high-power fields ( 100 cells) were counted. The proportion of core-positive cells were $62.5 \%, 51.5 \%, 49.2 \%$, and $61.7 \%$ for WT, R70Q, L91M, and R70QL91M, respectively, which was similar to that of the $\mathrm{JFH}-1$ strain, which was $70.0 \%$ (Fig. 1b). ${ }^{10}$

\section{Comparison of virus replication between core amino acid 70/91 substitutions}

After transfecting TPF1-M170T clones, intracellular HCV RNA was quantified by real-time PCR. The RNA replicationdefective mutant TPF1- $\triangle$ GDD kindly provided by Mori et al. (data in submission) was used as the negative control. The construct was created by in-frame deletion of the active-site motif GDD in NS5B polymerase. There were no significant differences in HCV RNA between TPF1-M170T clones and TPF1- $\triangle$ GDD (Fig. 2a).

Intracellular $\mathrm{HCV}$ core $\mathrm{Ag}$ was measured from extracted protein lysates to evaluate virus replication (Fig. 2b). The levels of intracellular HCV core $\mathrm{Ag}$ in each clone increased from day 1 to 5 , in contrast to the levels of TPF1- $\triangle G D D$, which gradually declined. These results indicate that these clones replicated and were efficiently synthesized inside cells. L91M demonstrated the highest HCV core Ag levels among the clones, but there were no significant differences in the core amino acid 70/91 substitutions.

\section{Comparison of protein synthesis between core amino acid 70/91 substitutions}

Virus replication was further confirmed by measuring HCV protein by Western blot analysis with anti-NS5A antibodies. The intensity of all clones was higher than that of the negative control (Fig. 3). The intensity of NS5A protein of the L91M clone was the highest among these clones, which corresponds to the results obtained from the HCV core Ag.

\section{Difference in IFN resistance between genotypes $1 b$ and $2 a$}

Sensitivity of the TPF1-M170T clones with core substitutions to IFN- $\alpha-2 b$ treatment was assessed (Fig. 4). IFN- $\alpha-2 b$ is clinically effective for HCV and was reported to have inhibitory effects on HCV replication in subgenomic replicons. ${ }^{19}$ Each clone was transfected into Huh7-ALS32.50 cells by electroporation, which were then seeded into 12-well dishes. Four 
a.

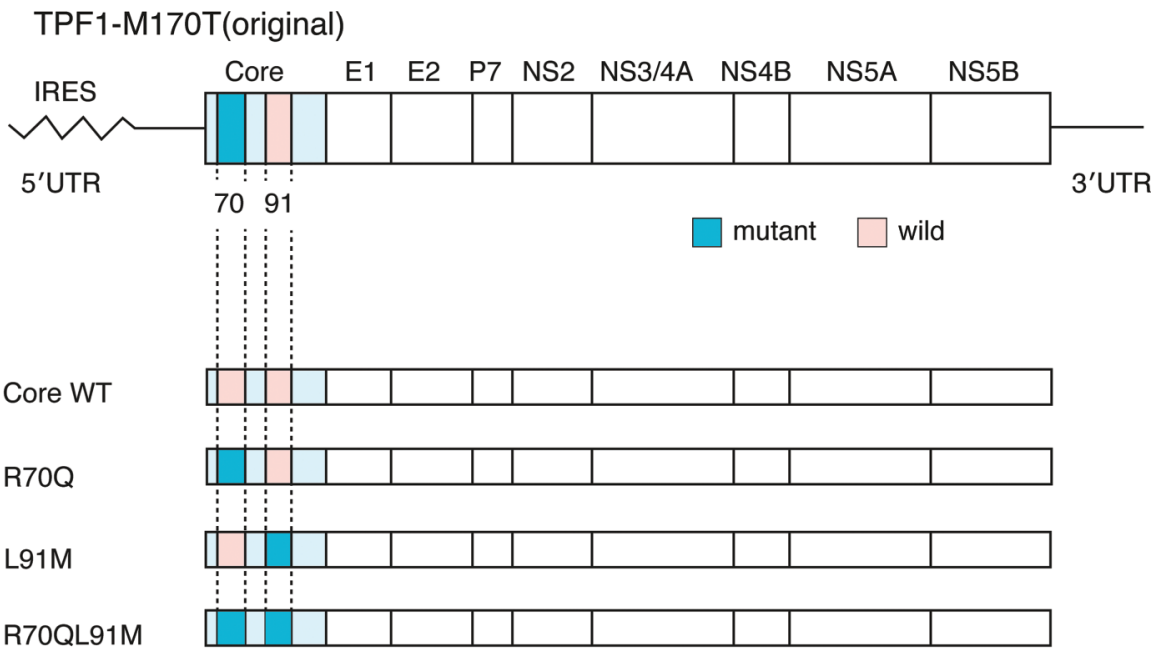

b.

Core WT

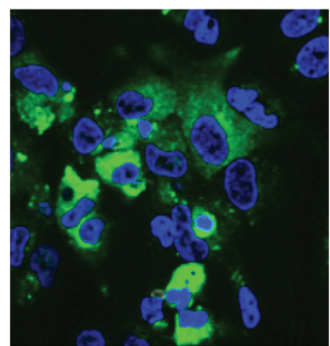

(68 cells / 102 cells) $62.5 \%$
$\mathrm{R} 70 \mathrm{Q}$

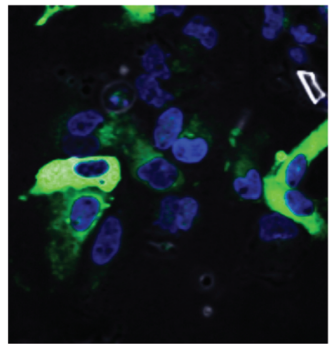

(53 cells $/ 109$ cells) $51.5 \%$
L91M

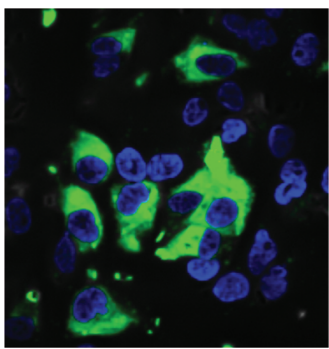

(93 cells $/ 189$ cells) $49.2 \%$
R70QL91M

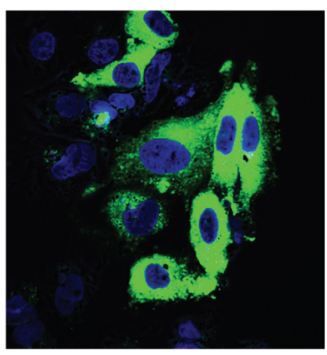

(84 cells $/ 137$ cells)

$61.7 \%$
JFH1

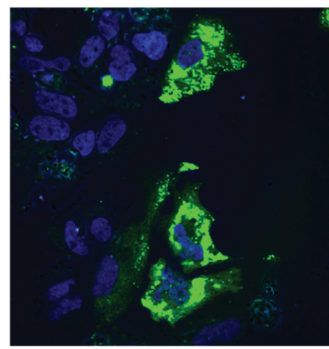

(60 cells $/ 85$ cells) $70 \%$

Fig. 1. Construction of TPF1-M170T clones and evaluation of transfection efficiency. (a) Genomic structures of the original pTPF1-M170T and their developed clones: Core WT, R70Q, L91M, and R70QL91M. As the pTPF1-M170T already harbors R70Q at the core region, the R70Q clone is the same as the original one. (b) Immunofluorescence assay of the developed clones. Hepatitis $\mathrm{C}$ virus-positive cells at $3 \mathrm{~d}$ post-transfection are visualized with anti-core antibody (green); nuclei are visualized with DAPI (blue) (magnification, $\times 60$ ).

days post-transfection, cells were cultured for $48 \mathrm{~h}$ in various concentrations of IFN- $\alpha-2 b$. Protein was extracted from the cells, and the level of HCV core Ag was quantified by CLIA.

JFH- 1 was the most sensitive to IFN- $\alpha-2 b$, with an $\mathrm{EC}_{50}$ of $1.41 \pm 1.29 \mathrm{IU} / \mathrm{mL}$. The values of $\mathrm{EC}_{50}$ for Core $\mathrm{WT}, \mathrm{R} 70 \mathrm{Q}$, and R70QL91M were $5.10 \pm 1.27 \mathrm{IU} / \mathrm{mL}, 3.23 \pm 0.37 \mathrm{IU} / \mathrm{mL}$, and $3.81 \pm 1.05 \mathrm{IU} / \mathrm{mL}$, respectively. L91M demonstrated resistance at an $\mathrm{EC}_{50}$ of $17.1 \pm 6.86 \mathrm{IU} / \mathrm{mL}$. Compared with the Core WT, L91M did not significantly differ in the level of $\mathrm{EC}_{50}$, but $\mathrm{JFH}-1$ did $(p<0.05)$, confirming that the $1 \mathrm{~b}$ clone was more resistant than the $2 \mathrm{a}$ clone.

\section{Discussion}

This study describes a novel cell culture system for HCV genotype $1 \mathrm{~b}$ with core amino acid 70/91 substitutions. This 
a Intracellular HCV RNA
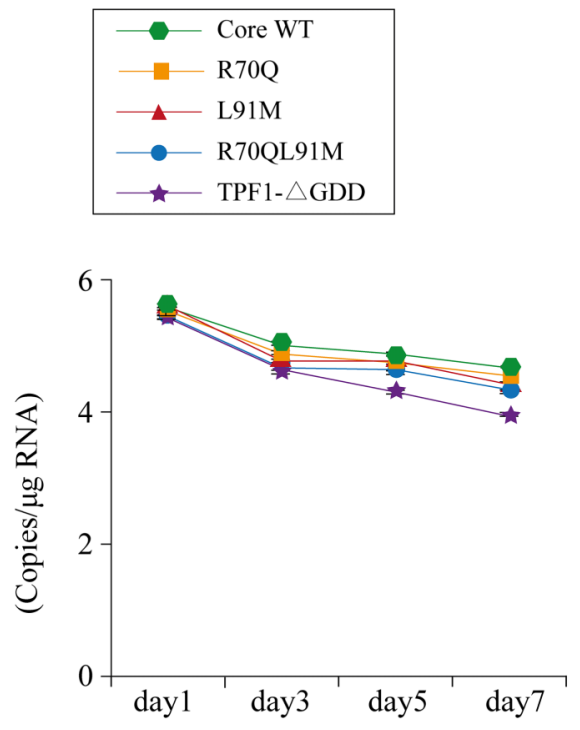

$\mathrm{b}$

\section{Intracellular HCV core Ag}
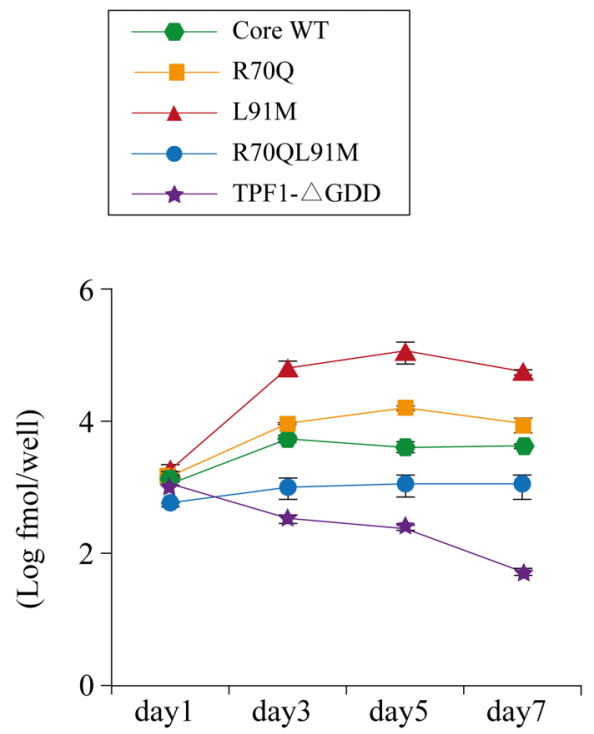

Fig. 2. Comparing replications of TPF1-M170T clones. (a) Time course of intracellular hepatitis $C$ virus (HCV)-RNA replications after in vitro-transcribed mutant and wild-type RNAs were transfected into Huh7-ALS32.50 cells. (b) Expression of intracellular HCV core antigen (Ag) as quantified by chemical reaction immunoassay. Data are presented as mean $\pm \mathrm{SD}$. Intracellular $\mathrm{HCV}$ core $\mathrm{Ag}$

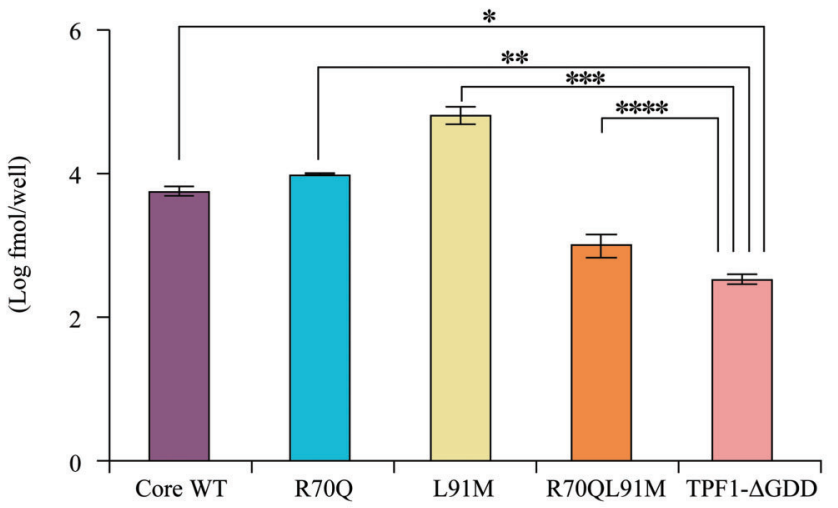

b

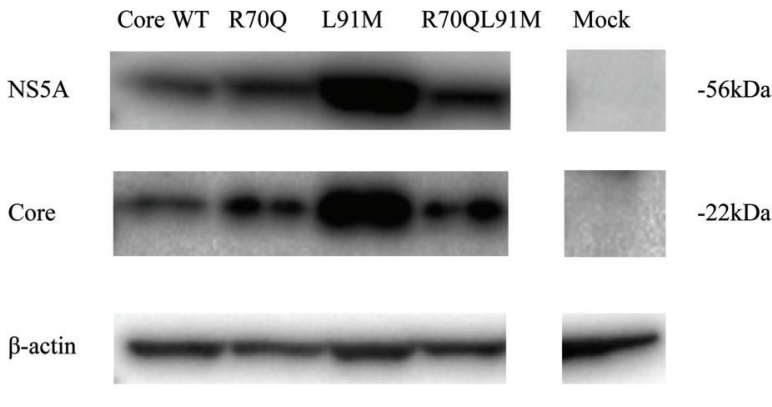

Fig. 3. Comparison of protein synthesis among the TPF1-M170T clones. (a) Comparison of intracellular hepatitis $\mathrm{C}$ virus $(\mathrm{HCV})$ core antigen $(\mathrm{Ag})$ at day 3. (b) Representative Western blot for NS5A and core proteins; ${ }^{*} p<0.05$. system could successfully demonstrate, for the first time, the IFN resistance of HCV genotype $1 \mathrm{~b}$ as clinically observed, after confirming $\mathrm{HCV}$ replication and translation. In this analysis, the TPF1- $\triangle$ GDD clone, which is the deletion mutant of the active site (GDD motif) of the NS5B polymerase, was used as a negative control. Although the quantity of intracellular HCV RNA in each of the clones tended to be higher than that of the control TPF1- $\triangle$ GDD clone, the differences were not statistically significant. One of the possible reasons is the low replication efficiency of the control clone. Even in the JFH-1, an evident difference with the GDD mutant does not emerge until approximately ten days after transfection. ${ }^{8}$ Moreover, an apparent difference in the genotype $1 \mathrm{~b}$ replicon was previously observed at only 72 hours post-transfection. ${ }^{20}$ Another possibility is that the amount of input HCV RNA exceeded the replicated RNA. To solve this problem, we measured HCV core $\mathrm{Ag}$, which is not influenced by input RNA, to verify higher production of $\mathrm{HCV}$ core $\mathrm{Ag}$ in each clone compared with TPF1- $\triangle$ GDD clone. Furthermore, this result was validated by Western blotting using anti-NS5A antibodies. We therefore concluded that our clone could be successfully transfected, replicated, and translated. Next, these clones were assessed for IFN resistance. The results demonstrate the IFN resistance of HCV genotype $1 \mathrm{~b}$. To the best of our knowledge, this is the first report that compared IFN sensitivity of HCV genotypes $1 \mathrm{~b}$ and $2 \mathrm{a}$ using the HCV cell culture system.

Compared with our previous results, the order of the replication efficiency of each clone was different. Funaoka et al. ${ }^{13}$ reported that $\mathrm{R} 70 \mathrm{Q}$ and $\mathrm{L} 91 \mathrm{M}$ replicated significantly better than the wild type, whereas Tasaka-Fujita et al. ${ }^{21}$ found that R70Q replicated less and L91M replicated more. The former report used genotype $2 a$, whereas the latter 
IFN-alpha $2 b$

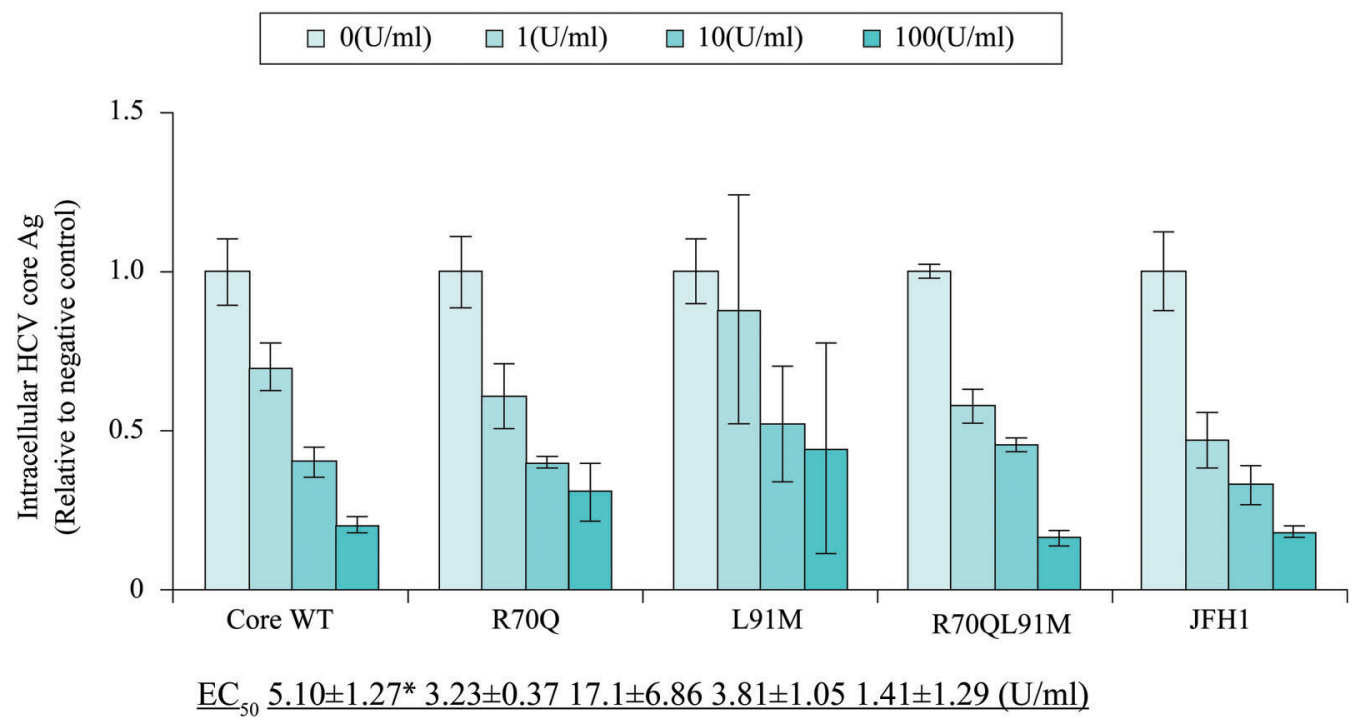

Fig. 4. Comparison of interferon (IFN) sensitivity between TPF1-M170T and JFH-1. Expression of intracellular HCV core Ag was measured by chemical reaction immunoassay at $72 \mathrm{~h}$ post-transfection of Core wild type (WT), R70Q, L91M, R70QL91M, and JFH-1. Cells were treated for $48 \mathrm{~h}$ with $0-100 \mathrm{IU} / \mathrm{mL}$. The values are displayed as percentages of the IFN-untreated control. $* p<0.05$ compared with JFH1.

used $1 \mathrm{~b} / 2 \mathrm{a}$ chimeric virus. As both reports used genotype 2a-derived sequences for NS proteins, which comprise a replication complex that is indispensable for virus replication, the differences in NS proteins may account for the contrasting results.

In this study, no significant difference in IFN response was observed between the core amino acid 70/91 substitutions, which is also in contrast to our previous report. ${ }^{13}$ One of the reasons may be the different replication efficiency of the JFH$1 \mathrm{HCV}$ cell culture, which enabled robust replication in the former study. The other possibility may be an extracellular IFN response that is different from intracellular mechanisms. However, in the study by Tasaka-Fujita et al., ${ }^{21}$ there was also no difference in IFN response between core 70/91 substitutions using $1 \mathrm{~b} / 2 \mathrm{a}$ chimeric clones. Nevertheless, a difference in the cell surface expression of MHC Class I in HCV-transfected cells was observed, which was considered a possible cause for IFN resistance. MHC class I is the molecule that is recognized by cytotoxic T cells to induce the elimination of infected cells. Therefore, when HCV hinders expression of MHC class I, infected cells may be able to continue replicating after the cessation of treatment. An alternative possibility concerns the type of cells used in the present study. IL28B SNPs in Huh7-ALS32.50 cells are all treatment-resistant heterotypes. Therefore, it might be difficult to find the difference between core amino acid 70/91 mutants and the wild type because of the pre-existing IFN-resistant environment. A different outcome may result if a treatment-sensitive cell line expressing the IL28B major homozygote is used, which would show a favorable response to exogenous IFN, whereas IFN-sensitive clones would be eliminated. In such a case, a significant difference may then be detectable. As it was clinically reported that a significant difference in treatment response among core substitutions was only observed in the patients with unfavorable IL28B SNPs, further experiments will be required to validate our results.

\section{Conclusions}

The results of this study from newly developed HCV cell culture system demonstrated, for the first time, that HCV genotype $1 \mathrm{~b}$ was significantly resistant to IFN treatment compared with genotype $2 \mathrm{a}$. This result indicates that this system reproduce clinical characteristics of HCV genotype $1 \mathrm{~b}$ in vitro and will be useful for developing novel antiviral chemotherapy against HCV.

\section{Acknowledgments}

The authors would like to thank Takaji Wakita for providing the pJFH1 full. This study was supported by grants from the Ministry of Education, Culture, Sports, Science and Technology-Japan, Japan Agency for Medical Research and Development, the Japan Society for the Promotion of Science, Ministry of Health, Labor Welfare-Japan, the Japan Health Science Foundation, and National Institute of Biomedical Innovation.

\section{Conflict of interest}

Dr. Asahina and Dr. Kakinuma belong to a donation-funded department funded by Chugai Pharmaceutical Co. Ltd., Toray Industries Inc., Bristol-Myers Squibb, Dainippon Sumitomo Pharma Co. Ltd., and Merck Sharp \& Dohme.

\section{Author contributions}

MT and MF contributed equally to this work.

\section{References}

[1] Global Burden Of Hepatitis C Working Group. Global burden of disease (GBD) for hepatitis C. J Clin Pharmacol 2004;44:20-29. doi: 10.1177/ 0091270003258669. 
[2] Jayasekera CR, Barry M, Roberts LR, Nguyen $\mathrm{MH}$. Treating hepatitis $\mathrm{C}$ in lower-income countries. N Engl J Med 2014;370:1869-1871. doi: 10.1056/ NEJMp1400160.

[3] Lozano R, Naghavi M, Foreman K, Lim S, Shibuya K, Aboyans V, etal. Global and regional mortality from 235 causes of death for 20 age groups in 1990 and 2010: a systematic analysis for the Global Burden of Disease Study 2010. Lancet 2012;380:2095-2128. doi: 10.1016/S0140-6736(12)61728-0.

[4] Manns MP1, McHutchison JG, Gordon SC, Rustgi VK, Shiffman M, Reindollar R, et $a l$. Peginterferon alfa-2b plus ribavirin compared with interferon alfa-2b plus ribavirin for initial treatment of chronic hepatitis $\mathrm{C}$ : a randomised trial. Lancet 2001;358:958-965. doi: 10.1016/S0140-6736(01)06102-5.

[5] Jacobson IM, McHutchison JG, Dusheiko G, Di Bisceglie AM, Reddy KR, Bzowej $\mathrm{NH}$, et al. Telaprevir for previously untreated chronic hepatitis $\mathrm{C}$ virus infection. N Engl J Med 2011;364:2405-2416. doi: 10.1056/NEJMoa1012912.

[6] Kumada H, Suzuki Y, Ikeda K, Toyota J, Karino Y, Chayama K, et al. Daclatasvir plus asunaprevir for chronic HCV genotype $1 \mathrm{~b}$ infection. Hepatology 2014;59:2083-2091. doi: 10.1002/hep.27113.

[7] Manns M, Pol S, Jacobson IM, Marcellin P, Gordon SC, Peng CY, et al. All-ora daclatasvir plus asunaprevir for hepatitis $C$ virus genotype $1 \mathrm{~b}$ : a multinational, phase 3, multicohort study. Lancet 2014;384:1576-1605. doi: 10. 1016/S0140-6736(14)61059-X.

[8] Blight KJ1, Kolykhalov AA, Rice CM. Efficient initiation of HCV RNA replication in cell culture. Science 2000;290:1972-1974. doi: 10.1126/science.290. 5498.1972.

[9] Lohmann V1, Körner F, Koch J, Herian U, Theilmann L, Bartenschlager R. Replication of subgenomic hepatitis $C$ virus RNAs in a hepatoma cell line. Science 1999;285:110-113. doi: 10.1126/science.285.5424.110.

[10] Wakita T, Pietschmann T, Kato T, Date T, Miyamoto M, Zhao Z, et al. Production of infectious hepatitis $C$ virus in tissue culture from a cloned viral genome. Nature Medicine 2005;11:791-796. doi: 10.1038/nm1268.

[11] Li YP1, Ramirez S, Jensen SB, Purcell RH, Gottwein JM, Bukh J. Highly efficient full-length hepatitis $C$ virus genotype 1 (strain TN) infectious culture system. Proc Natl Acad Sci U S A 2012;109:19757-19762. doi: 10.1073/pnas. 1218260109.

[12] Scheel TK1, Rice CM. Understanding the hepatitis $C$ virus life cycle paves the way for highly effective therapies. Nat Med 2013;19:837-849. doi: 10. $1038 / \mathrm{nm} .3248$.
[13] Funaoka Y, Sakamoto N, Suda G, Itsui Y, Nakagawa M, Kakinuma S, et al. Analysis of Interferon Signaling by Infectious Hepatitis C Virus Clones with Substitutions of Core Amino Acids 70 and 91. J Virol 2011;85:59865994. doi: 10.1128/JVI.02583-10.

[14] Yoshioka K, Kakumu S, Wakita T, Ishikawa T, Itoh $Y$, Takayanagi M, et al. Detection of hepatitis $C$ virus by polymerase chain reaction and response to interferon-alpha therapy:relationship to genotypes of hepatitis $C$ virus. Hepatology 1992;16:293-299. doi: 10.1002/hep.1840160203.

[15] Akuta N, Suzuki F, Kawamura Y, Yatsuji H, Sezaki H, Suzuki Y, et al. Amino acid substitutions in the hepatitis $\mathrm{C}$ virus core region are the important predictor of hepatocarcinogenesis. Hepatology 2007;46:1357-1364. doi: 10.1002/hep.21836.

[16] Akuta N, Suzuki F, Kawamura Y, Yatsuji H, Sezaki H, Suzuki Y, etal. Substitution of amino acid 70 in the hepatitis $C$ virus core region of genotype $1 \mathrm{~b}$ is an important predictor of elevated alpha-fetoprotein in patients without hepatocellular carcinoma. J Med Virol 2008;80:1354-1362. doi: 10.1002/jmv. 21202.

[17] Yokota T, Sakamoto N, Enomoto N, Tanabe Y, Miyagishi M, Maekawa S, et al. Inhibition of intracellular hepatitis $C$ virus replication by synthetic and vector-derived small interfering RNAs. EMBO Reports 2003;4:602-608. doi: 10.1038/ sj.embor.embor840.

[18] Tanabe Y, Sakamoto N, Enomoto N, Kurosaki M, Ueda E, Maekawa S, et al. Synergistic inhibition of intracellular hepatitis $C$ virus replication by combination of ribavirin and interferon-alpha. Journal of Infectious Diseases 2004;189:1129-1139. doi: 10.1086/382595.

[19] Fridell RA, Wang C, Sun JH, O'Boyle DR, Nower P, Valera L, et al. Genotypic and phenotypic analysis of variants resistant to hepatitis $C$ virus nonstructural protein 5A replication complex inhibitor BMS-790052 in humans: in vitro and in vivo correlations. Hepatology 2011;54:1924-1935. doi: 10 . 1002/hep.24594.

[20] Kanazawa N, Kurosaki M, Sakamoto N, Enomoto N, Itsui $Y$, Yamashiro T, et al. Journal of Virology 2004;78:9713-9720. doi: 10.1128/JVI.78.18.97139720.2004.

[21] Tasaka-Fujita, M, Sugiyama N, Kang W, Masaski T, Murayama A, Yamada N, et al. Amino Acid Polymorphisms in Hepatitis $C$ Virus Core Affect Infectious Virus Production and Major Histocompatibility Complex Class I Molecule Expression. Sci Rep 2015;5:139942015. doi: 10.1038/srep13994. 